\title{
Weighted Least-Squares Method for Designing Variable Fractional Delay 2-D FIR Digital Filters
}

\author{
Tian-Bo Deng, Senior Member, IEEE and Wu-Sheng Lu, Fellow, IEEE
}

\begin{abstract}
This paper proposes a closed-form weighted least-squares solution for designing variable two-dimensional (2-D) finite-impulse response (FIR) digital filters with continuously variable 2-D fractional delay responses. First, the coefficients of the variable 2-D transfer function are represented by using the polynomials of a pair of fractional delays $\left(p_{1}, p_{2}\right)$. Then the weighted squared-error function of the variable 2-D frequency response is derived without sampling the two frequencies $\left(\omega_{1}, \omega_{2}\right)$ and two fractional delays $\left(p_{1}, p_{2}\right)$, which leads to a significant reduction in computational complexity. With the assumption that the overall weighting function is separable and stepwise, the design problem is reduced to the minimization of the weighted squared-error function. Based on the error function, the closed-form optimal solutions for the coefficient matrices of the variable 2-D transfer function can be determined through solving a pair of matrix equations. In addition, Cholesky decomposition is applied to the final closed-form expressions in order to avoid some numerical instability problem. An example is given to illustrate the effectiveness of the proposed design method.
\end{abstract}

Index Terms-Two-dimensional digital filters, variable digital filters, variable fractional delay filters, weighted least-squares design.

\section{INTRODUCTION}

$\mathbf{I}$ $\mathrm{N}$ MANY signal processing applications and telecommunications, the frequency characteristics of digital filters need to be variable (adjustable). Such digital filters are referred to as "variable digital filters." Since digital filters have many advantages over the analog ones, especially in the variable case, digital filters are more flexible to implement, and research on the design and implementation of variable digital filters have received considerable attention. For simplicity, this paper will take the term "variable filter" to mean "variable digital filter." Generally speaking, variable filters can be classified basically into two main categories. The first one includes variable filters with only variable magnitude responses. For example, variable finite-impulse response (FIR) filters are designed for tuning magnitude responses in [1]-[4], and one-dimensional (1-D) infinite-impulse response (IIR) filters are investigated in [5], [6], where the coefficients of the recursive variable filters are expressed as the multi-dimensional (M-D) polynomials of the

Manuscript received January 1999; revised September 1999. The work of T.-B. Deng was supported by the Telecommunications Advancement Foundation (TAF) of Japan. The work of theW.-S. Lu was supported by the Natural Sciences and Engineering Research Council of Canada. This paper was recommended by Associate Editor J. Astola.

T.-B. Deng is with the Department of Information Science, Faculty of Science, Toho University, Funabashi, Chiba 274-8510, Japan.

W.-S. Lu is with the Department of Electrical and Computer Engineering, University of Victoria, Victoria, BC V8W 3P6, Canada.

Publisher Item Identifier S 1057-7130(00)01462-2. spectral parameters that control the magnitude characteristics of the variable filters. Since the stability of variable IIR filters should be guaranteed during the tuning process, one method for solving the stability problem has been proposed in [6]. The approaches can also be extended to the two-dimensional (2-D) case [7], [8]. To simplify the 2-D design problem, the decomposition-based approach is very efficient [9]. In the recent paper [10], most of the existing methods for designing variable filters in this category are surveyed and compared. This paper will not discuss the variable filters in this category, and the interested reader is referred to [10] for details. The second category with which we are concerned in this paper deals with the variable filters with only variable phase or group delay responses. The variable filters with variable fractional delay (FD) responses are extremely useful in applications such as timing adjustment in a digital modem [11], [12], music instrument modeling, audio signal processing [13], [14], incommensurate-ratio sampling rate conversion, and speech coding [15], since the group and phase delay time of this kind of variable filters can take values which are not necessarily multiples but fractions of the sample interval. So far, many design methods for this class of variable 1-D filters have been proposed. For example, Farrow proposed a polynomial-based method [16], which is very simple to design and implement. Paper [17] examines some methods of FIR designs including the Lagrangian interpolation method. Recently, weighted least-squares methods with and without discretizing parameters have been proposed to improve the design accuracy with reduced computational complexity [18], [19]. Paper [15] provides a comprehensive review and comparison of the existing methods for designing and implementing such 1-D variable filters. As mentioned in [15], variable FD filters have many potential applications in M-D digital signal processing fields. For example, variable three-dimensional (3-D) FD filters can be used in continuous frame interpolations of video data, and 2-D ones can be applied to the continuous subpixel interpolations of still images for image magnification and minification. In addition, 2-D FD filters based on piecewise polynomial interpolations have been investigated in the contex of image resampling; see, for example, [20]-[24] and the references therein. No matter what degrees of piecewise polynomials are used, these interpolation methods are aimed to approximate the ideal low-pass filter with cutoff frequency equal to Nyquist frequency in the frequency domain as accurately as possible.

In this paper, we propose a method for designing variable 2-D FIRFDfiltersinthefrequency domain directly sothatthefractional dealys of the variable filters in the passband are continuously adjustable. The basic idea is to represent the coefficients of the vari- 
able 2-D transfer function as the polynomials of the fractional delays $\left(p_{1}, p_{2}\right)$, and then derive a weighted squared-error function of variable 2-D frequency response without discretizing the 2-D frequencies $\left(\omega_{1}, \omega_{2}\right)$ and 2-D fractional delays $\left(p_{1}, p_{2}\right)$. Finally, closed-formexpressions for the optimal coefficientmatrices of the variable 2-D FIR filter are obtained by minimizing the error function. A significant advantage of the proposed method is that it does not need the discretizations of the parameters $\omega_{1}, \omega_{2}, p_{1}$, and $p_{2}$ to derive the weighted squared-error function; thus, the final design accuracy is not affected by the grid densities of the sampling points. Also, the discretization-free approach leads to a considerable reduction in computational complexity. An example is given to demonstrate that the method can achieve extremely accurate designs efficiently.

\section{Problem Formulation AND OBJECTIVE FunCtion}

The desired variable 2-D FD response is

$$
H_{d}\left(\omega_{1}, \omega_{2}, p_{1}, p_{2}\right)=e^{-j\left[\omega_{1}\left(D_{1}+p_{1}\right)+\omega_{2}\left(D_{2}+p_{2}\right)\right]}
$$

where $\left(\omega_{1}, \omega_{2}\right)$ are the normalized 2-D angular frequencies, $\omega_{1}$, $\omega_{2} \in[-\pi, \pi],\left(p_{1}, p_{2}\right)$ are the $2-\mathrm{D}$ variable fractional delays, $p_{1}$, $p_{2} \in[0,1]$, and $\left(D_{1}, D_{2}\right)$ are the positive integers that represent the integer parts of the total group delays. Obviously, $p_{1}$ and $p_{2}$ are continuously variable in the above ranges. Here, we want to use the variable FIR transfer function

$$
H\left(z_{1}, z_{2}, p_{1}, p_{2}\right)=H_{1}\left(z_{1}, p_{1}\right) H_{2}\left(z_{2}, p_{2}\right)
$$

to approximate the desired variable frequency response (1), where

$$
H_{1}\left(z_{1}, p_{1}\right)=\sum_{n_{1}=0}^{N_{1}} a_{1 n_{1}}\left(p_{1}\right) z_{1}^{-n_{1}}
$$

can be regarded as the 1-D transfer function with variable coefficients $a_{1 n_{1}}\left(p_{1}\right)$ which are the functions of $p_{1}$. The reason why the transfer function $H\left(z_{1}, z_{2}, p_{1}, p_{2}\right)$ in (2) assumes a separable form is that the desired frequency response $H_{d}\left(\omega_{1}, \omega_{2}, p_{1}, p_{2}\right)$ in (1) is separable. Like in [16], for the sake of generality and easy treatment, we also assume that $a_{1 n_{1}}\left(p_{1}\right)$ are the $1-\mathrm{D}$ polynomials of $p_{1}$, i.e.,

$$
a_{1 n_{1}}\left(p_{1}\right)=\sum_{k_{1}=0}^{K_{1}} a_{1}\left(n_{1}, k_{1}\right) p_{1}^{k_{1}} \text {. }
$$

Substituting (4) to (3) gives

$$
\begin{aligned}
H_{1}\left(z_{1}, p_{1}\right) & =\sum_{n_{1}=0}^{N_{1}} \sum_{k_{1}=0}^{K_{1}} a_{1}\left(n_{1}, k_{1}\right) z_{1}^{-n_{1}} p_{1}^{k_{1}} \\
& =\boldsymbol{z}_{1}^{T} \boldsymbol{A}_{1} \boldsymbol{p}_{1}
\end{aligned}
$$

where

$$
\begin{gathered}
\boldsymbol{z}_{1}^{T}=\left[\begin{array}{lllll}
1 & z_{1}^{-1} & z_{1}^{-2} & \cdots & z_{1}^{-N_{1}}
\end{array}\right] \\
A_{1}=\left[\begin{array}{cccc}
a_{1}(0,0) & a_{1}(0,1) & \cdots & a_{1}\left(0, K_{1}\right) \\
a_{1}(1,0) & a_{1}(1,1) & \cdots & a_{1}\left(1, K_{1}\right) \\
\vdots & \vdots & \vdots & \vdots \\
a_{1}\left(N_{1}, 0\right) & a_{1}\left(N_{1}, 1\right) & \cdots & a_{1}\left(N_{1}, K_{1}\right)
\end{array}\right] \\
\boldsymbol{p}_{1}=\left[\begin{array}{lllll}
1 & p_{1} & p_{1}^{2} & \cdots & p_{1}^{K_{1}}
\end{array}\right]^{T} .
\end{gathered}
$$

Similarly, we can express $H_{2}\left(z_{2}, p_{2}\right)$ as

$$
\begin{aligned}
H_{2}\left(z_{2}, p_{2}\right) & =\sum_{n_{2}=0}^{N_{2}} \sum_{k_{2}=0}^{K_{2}} a_{2}\left(n_{2}, k_{2}\right) z_{2}^{-n_{2}} p_{2}^{k_{2}} \\
& =z_{2}^{T} \boldsymbol{A}_{2} \boldsymbol{p}_{2}
\end{aligned}
$$

where

$$
\begin{aligned}
z_{2}^{T} & =\left[\begin{array}{ccccc}
1 & z_{2}^{-1} & z_{2}^{-2} & \cdots & z_{2}^{-N_{2}}
\end{array}\right] \\
\boldsymbol{A}_{2} & =\left[\begin{array}{cccc}
a_{2}(0,0) & a_{2}(0,1) & \cdots & a_{2}\left(0, K_{2}\right) \\
a_{2}(1,0) & a_{2}(1,1) & \cdots & a_{2}\left(1, K_{2}\right) \\
\vdots & \vdots & \vdots & \vdots \\
a_{2}\left(N_{2}, 0\right) & a_{2}\left(N_{2}, 1\right) & \cdots & a_{2}\left(N_{2}, K_{2}\right)
\end{array}\right] \\
\boldsymbol{p}_{2} & =\left[\begin{array}{lllll}
1 & p_{2} & p_{2}^{2} & \cdots & p_{2}^{K}
\end{array}\right]^{T} .
\end{aligned}
$$

The orders $K_{1}$ in (4) and $K_{2}$ in (9) are chosen by the designer to produce an FD filter with satisfactory performance and manageable computational complexity. High orders $\left(K_{1}, K_{2}\right)$ yield improved design with increased computation cost at the design as well as implementation stages, while low orders $\left(K_{1}, K_{2}\right)$ lead to inexpensive design and implementation but degraded performance.

The integer parts of the total group delays in (1), namely $D_{1}$ and $D_{2}$, are chosen as

$$
D_{i}= \begin{cases}\frac{N_{i}-1}{2}, & \text { for odd } N_{i} \\ \frac{N_{i}}{2}, & \text { for even } N_{i}\end{cases}
$$

where $i=1,2$. This is because we want to use the transfer function (2) to approximate the desired frequency response (1), which is linear phase. Therefore, the variable 2-D transfer function (2) can be expressed as

$$
H\left(z_{1}, z_{2}, p_{1}, p_{2}\right)=z_{1}^{T} \boldsymbol{A}_{1} \boldsymbol{p}_{1} z_{2}^{T} A_{2} \boldsymbol{p}_{2}
$$

whose frequency response is given by

$$
H\left(\omega_{1}, \omega_{2}, p_{1}, p_{2}\right)=\boldsymbol{\omega}_{1}^{T} \boldsymbol{A}_{1} \boldsymbol{p}_{1} \boldsymbol{\omega}_{2}^{T} \boldsymbol{A}_{2} \boldsymbol{p}_{2}
$$

where

$$
\begin{aligned}
\boldsymbol{\omega}_{1}^{T} & =\left[\begin{array}{lllll}
1 & e^{-j \omega_{1}} & e^{-j 2 \omega_{1}} & \cdots & e^{-j N_{1} \omega_{1}}
\end{array}\right] \\
\boldsymbol{\omega}_{2}^{T} & =\left[\begin{array}{lllll}
1 & e^{-j \omega_{2}} & e^{-j 2 \omega_{2}} & \cdots & e^{-j N_{2} \omega_{2}}
\end{array}\right] .
\end{aligned}
$$

Defining the complex-valued error

$$
\begin{aligned}
e\left(\omega_{1}, \omega_{2}, p_{1}, p_{2}\right)= & H\left(\omega_{1}, \omega_{2}, p_{1}, p_{2}\right) \\
& -H_{d}\left(\omega_{1}, \omega_{2}, p_{1}, p_{2}\right)
\end{aligned}
$$

our objective here is to find the optimal coefficient matrices $A_{1}$ and $A_{2}$ such that the total weighted squared-error

$$
\begin{array}{r}
J\left(\boldsymbol{A}_{1}, \boldsymbol{A}_{2}\right)=\int_{\omega_{1}=-\pi}^{\pi} \int_{\omega_{2}=-\pi}^{\pi} \int_{p_{1}=0}^{1} \int_{p_{2}=0}^{1} W\left(\omega_{1}, \omega_{2}, p_{1}, p_{2}\right) \\
\cdot\left|e\left(\omega_{1}, \omega_{2}, p_{1}, p_{2}\right)\right|^{2} d \omega_{1} d \omega_{2} d p_{1} d p_{2}
\end{array}
$$


is minimized, where $W\left(\omega_{1}, \omega_{2}, p_{1}, p_{2}\right)$ is a nonnegative fourdimensional weighting function. In this paper, we assume that the weighting function is separable, i.e.,

$$
W\left(\omega_{1}, \omega_{2}, p_{1}, p_{2}\right)=W_{1}\left(\omega_{1}\right) W_{2}\left(\omega_{2}\right) W_{3}\left(p_{1}\right) W_{4}\left(p_{2}\right)
$$

and the 1-D functions $W_{1}\left(\omega_{1}\right), W_{2}\left(\omega_{2}\right), W_{3}\left(p_{1}\right), W_{4}\left(p_{2}\right)$ are piecewise constant. For example, for the frequencies $\omega_{1}$ in the range $[0, \pi]$, we first divide the interval $[0, \pi]$ into the union of several small intervals (sub-intervals) as

$$
\begin{aligned}
{[0, \pi]=\left[0, \omega_{1}(1)\right] \cup\left[\omega_{1}(1), \omega_{1}(2)\right] } & \cup \cdots \\
& \cup\left[\omega_{1}\left(L_{1}-1\right), \omega_{1}\left(L_{1}\right)\right]
\end{aligned}
$$

where $\omega_{1}\left(L_{1}\right)=\pi$. Then, the sub-weighting function $W_{1}\left(\omega_{1}\right)$ is chosen to be constant in each sub-interval, i.e.,

$$
W_{1}\left(\omega_{1}\right)=r_{1 l_{1}}, \quad \text { for } \quad \omega_{1} \in\left[\omega_{1}\left(l_{1}-1\right), \omega_{1}\left(l_{1}\right)\right]
$$

where $r_{1 l_{1}}$ are constants for $l_{1}=1,2, \cdots, L_{1}$. In addition, we assume that the sub-weighting function $W_{1}\left(\omega_{1}\right)$ for $\omega_{1} \in$ $[-\pi, 0]$ is symmetric with respect to $\omega_{1}=0$. Likely, $W_{2}\left(\omega_{2}\right)$ is defined as

$$
W_{2}\left(\omega_{2}\right)=r_{2 l_{2}}, \quad \text { for } \quad \omega_{2} \in\left[\omega_{2}\left(l_{2}-1\right), \omega_{2}\left(l_{2}\right)\right]
$$

where $l_{2}=1,2, \cdots, L_{2}$. As for the sub-weighting function $W_{3}\left(p_{1}\right)$, the interval $[0,1]$ is divided into the union of sub-intervals as

$[0,1]=\left[0, p_{1}(1)\right] \cup\left[p_{1}(1), p_{1}(2)\right] \cup \cdots \cup\left[p_{1}\left(M_{1}-1\right), p_{1}\left(M_{1}\right)\right]$

where $p_{1}\left(M_{1}\right)=1$. For each sub-interval, the corresponding sub-weighting function $W_{3}\left(p_{1}\right)$ is constant, i.e.,

$$
W_{3}\left(p_{1}\right)=r_{3 m_{1}}, \quad \text { for } \quad p_{1} \in\left[p_{1}\left(m_{1}-1\right), p_{1}\left(m_{1}\right)\right]
$$

where $m_{1}=1,2, \cdots, M_{1}$. Similarly, $W_{4}\left(p_{2}\right)$ can be defined as

$$
W_{4}\left(p_{2}\right)=r_{4 m_{2}}, \quad \text { for } \quad p_{2} \in\left[p_{2}\left(m_{2}-1\right), p_{2}\left(m_{2}\right)\right]
$$

where $m_{2}=1,2, \cdots, M_{2}$. Note that

$$
\begin{aligned}
\left|e\left(\omega_{1}, \omega_{2}, p_{1}, p_{2}\right)\right|^{2}= & e\left(\omega_{1}, \omega_{2}, p_{1}, p_{2}\right) e^{*}\left(\omega_{1}, \omega_{2}, p_{1}, p_{2}\right) \\
= & {\left[H\left(\omega_{1}, \omega_{2}, p_{1}, p_{2}\right)-H_{d}\left(\omega_{1}, \omega_{2}, p_{1}, p_{2}\right)\right] } \\
& \cdot\left[H^{*}\left(\omega_{1}, \omega_{2}, p_{1}, p_{2}\right)-H_{d}^{*}\left(\omega_{1}, \omega_{2}, p_{1}, p_{2}\right)\right]
\end{aligned}
$$

with

$$
\begin{aligned}
H^{*}\left(\omega_{1}, \omega_{2}, p_{1}, p_{2}\right) & =\bar{\omega}_{1}^{T} \boldsymbol{A}_{1} \boldsymbol{p}_{1} \overline{\boldsymbol{\omega}}_{2}^{T} \boldsymbol{A}_{2} \boldsymbol{p}_{2} \\
& =\left[\overline{\boldsymbol{\omega}}_{1}^{T} \boldsymbol{A}_{1} \boldsymbol{p}_{1} \overline{\boldsymbol{\omega}}_{2}^{T} \boldsymbol{A}_{2} \boldsymbol{p}_{2}\right]^{T} \\
& =\boldsymbol{p}_{2}^{T} \boldsymbol{A}_{2}^{T} \overline{\boldsymbol{\omega}}_{2} \boldsymbol{p}_{1}^{T} \boldsymbol{A}_{1}^{T} \overline{\boldsymbol{\omega}}_{1}
\end{aligned}
$$

where $\bar{\omega}_{1}=\omega_{1}^{*}, \bar{\omega}_{2}=\omega_{2}^{*}$, and [.] ${ }^{*}$ means the complex-conjugate of [•]. Substituting (1), (15), and (26) into (25), we obtain

$$
\begin{aligned}
\left|e\left(\omega_{1}, \omega_{2}, p_{1}, p_{2}\right)\right|^{2} & =\omega_{1}^{T} \boldsymbol{A}_{1} \boldsymbol{p}_{1} \omega_{2}^{T} \boldsymbol{A}_{2} \boldsymbol{p}_{2} \boldsymbol{p}_{2}^{T} \boldsymbol{A}_{2}^{T} \overline{\boldsymbol{\omega}}_{2} \boldsymbol{p}_{1}^{T} \boldsymbol{A}_{1}^{T} \overline{\boldsymbol{\omega}}_{1} \\
& -2 \operatorname{Re}\left[\boldsymbol{\omega}_{1}^{T} \boldsymbol{A}_{1} \boldsymbol{p}_{1} \boldsymbol{\omega}_{2}^{T} \boldsymbol{A}_{2} \boldsymbol{p}_{2}\right. \\
& \left.\cdot H_{d}^{*}\left(\omega_{1}, \omega_{2}, p_{1}, p_{2}\right)\right]+1 \\
& =\left(\boldsymbol{\omega}_{1}^{T} \boldsymbol{A}_{1} \boldsymbol{p}_{1} \boldsymbol{p}_{1}^{T} \boldsymbol{A}_{1}^{T} \overline{\boldsymbol{\omega}}_{1}\right)\left(\boldsymbol{\omega}_{2}^{T} \boldsymbol{A}_{2} \boldsymbol{p}_{2} \boldsymbol{p}_{2}^{T} \boldsymbol{A}_{2}^{T} \overline{\boldsymbol{\omega}}_{2}\right) \\
& -2 \operatorname{Re}\left\{\boldsymbol{\omega}_{1}^{T} \boldsymbol{A}_{1} \boldsymbol{p}_{1} \omega_{2}^{T} \boldsymbol{A}_{2} \boldsymbol{p}_{2}\right. \\
& \left.\cdot e^{j\left[\omega_{1}\left(D_{1}+p_{1}\right)+\omega_{2}\left(D_{2}+p_{2}\right)\right]}\right\}+1
\end{aligned}
$$

Substituting (18) and (27) into (17), we obtain

$$
J\left(\boldsymbol{A}_{1}, \boldsymbol{A}_{2}\right)=J_{1}\left(A_{1}, \boldsymbol{A}_{2}\right)+J_{2}\left(\boldsymbol{A}_{1}, \boldsymbol{A}_{2}\right)+J_{3}
$$

By using the matrix property $\operatorname{tr}(A B)=\operatorname{tr}(B A)$ [25], where $\operatorname{tr}(\cdot)$ denotes the trace of the matrix involved, $J_{1}\left(\boldsymbol{A}_{1}, \boldsymbol{A}_{2}\right)$ in (28) can be expressed as

$$
\begin{aligned}
& J_{1}\left(\boldsymbol{A}_{1}, \boldsymbol{A}_{2}\right)=\int_{\omega_{1}} \int_{\omega_{2}} \int_{p_{1}} \int_{p_{2}} W_{1}\left(\omega_{1}\right) W_{2}\left(\omega_{2}\right) W_{3}\left(p_{1}\right) W_{4}\left(p_{2}\right) \\
& \cdot\left(\boldsymbol{\omega}_{1}^{T} A_{1} \boldsymbol{p}_{1} \boldsymbol{p}_{1}^{T} \boldsymbol{A}_{1}^{T} \overline{\boldsymbol{\omega}}_{1}\right)\left(\boldsymbol{\omega}_{2}^{T} A_{2} \boldsymbol{p}_{2} \boldsymbol{p}_{2}^{T} \boldsymbol{A}_{2}^{T} \overline{\boldsymbol{\omega}}_{2}\right) \\
& d \omega_{1} d \omega_{2} d p_{1} d p_{2} \\
& =\left[\int_{\omega_{1}} \int_{p_{1}} W_{1}\left(\omega_{1}\right) W_{3}\left(p_{1}\right)\right. \\
& \left.\cdot \omega_{1}^{T} \boldsymbol{A}_{1} \boldsymbol{p}_{1} \boldsymbol{p}_{1}^{T} A_{1}^{T} \bar{\omega}_{1} d \omega_{1} d p_{1}\right] \\
& \text {. }\left[\int_{\omega_{2}} \int_{p_{2}} W_{2}\left(\omega_{2}\right) W_{4}\left(p_{2}\right)\right. \\
& \left.\cdot \omega_{2}^{T} \boldsymbol{A}_{2} \boldsymbol{p}_{2} \boldsymbol{p}_{2}^{T} A_{2}^{T} \bar{\omega}_{2} d \omega_{2} d p_{2}\right] \\
& =\operatorname{tr}\left[\int_{\omega_{1}} \int_{p_{1}} W_{1}\left(\omega_{1}\right) W_{3}\left(p_{1}\right) \boldsymbol{p}_{1} \boldsymbol{p}_{1}^{T}\right. \\
& \left.\cdot A_{1}^{T} \bar{\omega}_{1} \omega_{1}^{T} \boldsymbol{A}_{1} d \omega_{1} d p_{1}\right] \\
& \times \operatorname{tr}\left[\int_{\omega_{2}} \int_{p_{2}} W_{2}\left(\omega_{2}\right) W_{4}\left(p_{2}\right) \boldsymbol{p}_{2} \boldsymbol{p}_{2}^{T}\right. \\
& \text { - } \left.A_{2}^{T} \bar{\omega}_{2} \omega_{2}^{T} \boldsymbol{A}_{2} d \omega_{2} d p_{2}\right] \\
& =\operatorname{tr}\left[\int_{p_{1}} W_{3}\left(p_{1}\right) \boldsymbol{p}_{1} \boldsymbol{p}_{1}^{T} d p_{1} \cdot \boldsymbol{A}_{1}^{T}\right. \\
& \left.\int_{\omega_{1}} W_{1}\left(\omega_{1}\right) \bar{\omega}_{1} \omega_{1}^{T} d \omega_{1} \cdot \boldsymbol{A}_{1}\right] \\
& \times \operatorname{tr}\left[\int_{p_{2}} W_{4}\left(p_{2}\right) \boldsymbol{p}_{2} \boldsymbol{p}_{2}^{T} d p_{2} \cdot \boldsymbol{A}_{2}^{T}\right. \\
& \left.\cdot \int_{\omega_{2}} W_{2}\left(\omega_{2}\right) \bar{\omega}_{2} \boldsymbol{\omega}_{2}^{T} d \omega_{2} \cdot \boldsymbol{A}_{2}\right] \\
& =\operatorname{tr}\left(\boldsymbol{P}_{1} \boldsymbol{A}_{1}^{T} \boldsymbol{\Omega}_{1} A_{1}\right) \operatorname{tr}\left(\boldsymbol{P}_{2} \boldsymbol{A}_{2}^{T} \boldsymbol{\Omega}_{2} \boldsymbol{A}_{2}\right)
\end{aligned}
$$

where

$$
\boldsymbol{P}_{1}=\int_{0}^{1} W_{3}\left(p_{1}\right) \boldsymbol{p}_{1} \boldsymbol{p}_{1}^{T} d p_{1}=\sum_{m_{1}=1}^{M_{1}} r_{3 m_{1}} \boldsymbol{P}_{1 m_{1}}
$$

$$
\begin{aligned}
\boldsymbol{P}_{1 m_{1}}= & \int_{p_{1}\left(m_{1}-1\right)}^{p_{1}\left(m_{1}\right)} \\
& \cdot\left[\begin{array}{ccccc}
1 & p_{1} & p_{1}^{2} & \cdots & p_{1}^{K_{1}} \\
p_{1} & p_{1}^{2} & p_{1}^{3} & \cdots & p_{1}^{K_{1}+1} \\
\vdots & \vdots & \vdots & \cdots & \vdots \\
p_{1}^{K_{1}} & p_{1}^{K_{1}+1} & p_{1}^{K_{1}+2} & \cdots & p_{1}^{2 K_{1}}
\end{array}\right] d p_{1} \\
= & {\left[P_{1 m_{1}}(i, j)\right] }
\end{aligned}
$$


and $P_{1 m_{1}}(i, j)$ is the $(i, j)$ th entry of the matrix $\boldsymbol{P}_{1 m_{1}}$, which can be written as

$$
\begin{gathered}
P_{1 m_{1}}(i, j)=\frac{p_{1}^{k}\left(m_{1}\right)-p_{1}^{k}\left(m_{1}-1\right)}{k} ; \quad i=1,2, \cdots,\left(K_{1}+1\right) ; \\
\begin{array}{l}
j=1,2, \cdots,\left(K_{1}+1\right) \\
k=i+j-1
\end{array}
\end{gathered}
$$

In addition, the matrix $\Omega_{1}$ in (29) is given by

$$
\Omega_{1}=\int_{-\pi}^{\pi} W_{1}\left(\omega_{1}\right) \bar{\omega}_{1} \omega_{1}^{T} d \omega_{1}=2 \sum_{l_{1}=1}^{L_{1}} r_{1 l_{1}} \Omega_{1 l_{1}}
$$

where we have (34), shown at the bottom of the page, with

$$
\begin{array}{r}
\Omega_{1 l_{1}}(i, j) \\
\quad= \begin{cases}\omega_{1}\left(l_{1}\right)-\omega_{1}\left(l_{1}-1\right), & \text { for } k=0 \\
\frac{\sin \left[k \omega_{1}\left(l_{1}\right)\right]-\sin \left[k \omega_{1}\left(l_{1}-1\right)\right]}{k}, & \text { for } k \neq 0\end{cases}
\end{array}
$$

being the $(i, j)$ th entry of the matrix $\Omega_{1 l_{1}}$, and $i, j=$ $1,2, \cdots,\left(N_{1}+1\right), k=i-j$. Obviously, $\Omega_{1 l_{1}}$ are Toeplitz matrices, hence $\Omega_{1}$ is also a Toeplitz matrix. Matrices $\boldsymbol{P}_{2}$ and $\Omega_{2}$ can be evaluated in a similar manner.

In the second term of (27)

$$
\begin{aligned}
& \boldsymbol{\omega}_{1}^{T} \boldsymbol{A}_{1} \boldsymbol{p}_{1} \boldsymbol{\omega}_{2}^{T} \boldsymbol{A}_{2} \boldsymbol{p}_{2} \cdot e^{j\left[\omega_{1}\left(D_{1}+p_{1}\right)+\omega_{2}\left(D_{2}+p_{2}\right)\right]} \\
& \quad=\left[\boldsymbol{\omega}_{1}^{T} e^{j \omega_{1}\left(D_{1}+p_{1}\right)}\right] \boldsymbol{A}_{1} \boldsymbol{p}_{1}\left[\boldsymbol{\omega}_{2}^{T} e^{j \omega_{2}\left(D_{2}+p_{2}\right)}\right] \boldsymbol{A}_{2} \boldsymbol{p}_{2} \\
& \quad=\left(\boldsymbol{c}_{1}^{T}+j \boldsymbol{s}_{1}^{T}\right) \boldsymbol{A}_{1} \boldsymbol{p}_{1}\left(\boldsymbol{c}_{2}^{T}+j \boldsymbol{s}_{2}^{T}\right) \boldsymbol{A}_{2} \boldsymbol{p}_{2}
\end{aligned}
$$

thus

$$
\begin{aligned}
\operatorname{Re}\left\{\boldsymbol{\omega}_{1}^{T} A_{1} \boldsymbol{p}_{1} \boldsymbol{\omega}_{2}^{T} A_{2} \boldsymbol{p}_{2} \cdot e^{j\left[\omega_{1}\left(D_{1}+p_{1}\right)+\omega_{2}\left(D_{2}+p_{2}\right)\right]}\right\} & \\
= & \boldsymbol{c}_{1}^{T} \boldsymbol{A}_{1} \boldsymbol{p}_{1} \boldsymbol{c}_{2}^{T} \boldsymbol{A}_{2} \boldsymbol{p}_{2}-\boldsymbol{s}_{1}^{T} \boldsymbol{A}_{1} \boldsymbol{p}_{1} \boldsymbol{s}_{2}^{T} \boldsymbol{A}_{2} \boldsymbol{p}_{2}
\end{aligned}
$$

where we have (38), shown at the bottom of the page. Therefore, using the matrix property $\operatorname{tr}(\boldsymbol{A B})=\operatorname{tr}(\boldsymbol{B} \boldsymbol{A}), J_{2}\left(\boldsymbol{A}_{1}, \boldsymbol{A}_{2}\right)$ in (28) can be evaluated as

$$
\begin{aligned}
J_{2}\left(A_{1}, A_{2}\right) & \\
= & \int_{\omega_{1}} \int_{\omega_{2}} \int_{p_{1}} \int_{p_{2}} W_{1}\left(\omega_{1}\right) W_{2}\left(\omega_{2}\right) \\
& \cdot W_{3}\left(p_{1}\right) W_{4}\left(p_{2}\right)\left(\boldsymbol{c}_{1}^{T} A_{1} \boldsymbol{p}_{1} c_{2}^{T} A_{2} \boldsymbol{p}_{2}\right. \\
& \left.-\boldsymbol{s}_{1}^{T} \boldsymbol{A}_{1} \boldsymbol{p}_{1} \boldsymbol{s}_{2}^{T} \boldsymbol{A}_{2} \boldsymbol{p}_{2}\right) d \omega_{1} d \omega_{2} d p_{1} d p_{2}
\end{aligned}
$$

$$
\begin{aligned}
&= \int_{\omega_{1}} \int_{p_{1}} W_{1}\left(\omega_{1}\right) W_{3}\left(p_{1}\right) \boldsymbol{c}_{1}^{T} \boldsymbol{A}_{1} \boldsymbol{p}_{1} d \omega_{1} d p_{1} \\
& \cdot \int_{\omega_{2}} \int_{p_{2}} W_{2}\left(\omega_{2}\right) W_{4}\left(p_{2}\right) \boldsymbol{c}_{2}^{T} \boldsymbol{A}_{2} \boldsymbol{p}_{2} d \omega_{2} d p_{2} \\
&-\int_{\omega_{1}} \int_{p_{1}} W_{1}\left(\omega_{1}\right) W_{3}\left(p_{1}\right) \boldsymbol{s}_{1}^{T} \boldsymbol{A}_{1} \boldsymbol{p}_{1} d \omega_{1} d p_{1} \\
& \cdot \int_{\omega_{2}} \int_{p_{2}} W_{2}\left(\omega_{2}\right) W_{4}\left(p_{2}\right) \boldsymbol{s}_{2}^{T} \boldsymbol{A}_{2} \boldsymbol{p}_{2} d \omega_{2} d p_{2} \\
&=\left.\operatorname{tr} \int_{\omega_{1}} \int_{p_{1}} W_{1}\left(\omega_{1}\right) W_{3}\left(p_{1}\right) \boldsymbol{p}_{1} \boldsymbol{c}_{1}^{T} d \omega_{1} d p_{1} \cdot \boldsymbol{A}_{1}\right] \\
& \cdot \operatorname{tr}\left[\int_{\omega_{2}} \int_{p_{2}} W_{3}\left(\omega_{2}\right) W_{4}\left(p_{2}\right) \boldsymbol{p}_{2} \boldsymbol{c}_{2}^{T} d \omega_{2} d p_{2} \cdot \boldsymbol{A}_{2}\right] \\
&-\operatorname{tr}\left[\int_{\omega_{1}} \int_{p_{1}} W_{1}\left(\omega_{1}\right) W_{3}\left(p_{1}\right) \boldsymbol{p}_{1} \boldsymbol{s}_{1}^{T} d \omega_{1} d p_{1} \cdot \boldsymbol{A}_{1}\right] \\
& \cdot \operatorname{tr}\left[\int_{\omega_{2}} \int_{p_{2}} W_{3}\left(\omega_{2}\right) W_{4}\left(p_{2}\right) \boldsymbol{p}_{2} \boldsymbol{s}_{2}^{T} d \omega_{2} d p_{2} \cdot \boldsymbol{A}_{2}\right] \\
&=\operatorname{tr}\left(\boldsymbol{U}_{1} \boldsymbol{A}_{1}\right) \operatorname{tr}\left(\boldsymbol{U}_{2} \boldsymbol{A}_{2}\right)-\operatorname{tr}\left(\boldsymbol{V}_{1} \boldsymbol{A}_{1}\right) \operatorname{tr}\left(\boldsymbol{V}_{2} \boldsymbol{A}_{2}\right)
\end{aligned}
$$

where

$$
\begin{aligned}
\boldsymbol{U}_{1} & =\int_{-\pi}^{\pi} \int_{0}^{1} W_{1}\left(\omega_{1}\right) W_{3}\left(p_{1}\right) \boldsymbol{p}_{1} \boldsymbol{c}_{1}^{T} d \omega_{1} d p_{1} \\
& =2 \sum_{l_{1}=1}^{L_{1}} \sum_{m_{1}=1}^{M_{1}} r_{1 l_{1}} r_{3 m_{1}} \boldsymbol{U}_{1 l_{1} m_{1}}
\end{aligned}
$$

and

$$
\begin{aligned}
\boldsymbol{U}_{1 l_{1} m_{1}} & =\int_{\omega_{1}\left(l_{1}-1\right)}^{\omega_{1}\left(l_{1}\right)} \int_{p_{1}\left(m_{1}-1\right)}^{p_{1}\left(m_{1}\right)} \boldsymbol{p}_{1} c_{1}^{T} d \omega_{1} d p_{1} \\
& =\left[U_{1 l_{1} m_{1}}(i, j)\right] .
\end{aligned}
$$

In (41)

$$
U_{1 l_{1} m_{1}}(i, j)=\int_{p_{1}\left(m_{1}-1\right)}^{p_{1}\left(m_{1}\right)} p_{1}^{(i-1)} q_{1}\left(p_{1}\right) d p_{1}
$$

is the $(i, j)$ th entry of the matrix $\boldsymbol{U}_{1 l_{1} m_{1}}$, and

$$
q_{1}\left(p_{1}\right)= \begin{cases}\omega_{1}\left(l_{1}\right)-\omega_{1}\left(l_{1}-1\right), & \text { for } k=0 \\ \frac{\sin \left[k \omega_{1}\left(l_{1}\right)\right]-\sin \left[k \omega_{1}\left(l_{1}-1\right)\right]}{k}, & \text { for } k \neq 0\end{cases}
$$

where $k=D_{1}+p_{1}-j+1$. The integral (42) can be calculated by using fast and reliable numerical integration methods such

$$
\Omega_{1 l_{1}}=\int_{\omega_{1}\left(l_{1}-1\right)}^{\omega_{1}\left(l_{1}\right)}\left[\begin{array}{ccccc}
1 & \cos \omega_{1} & \cos 2 \omega_{1} & \cdots & \cos N_{1} \omega_{1} \\
\cos \omega_{1} & 1 & \cos \omega_{1} & \cdots & \cos \left(N_{1}-1\right) \omega_{1} \\
\cos 2 \omega_{1} & \cos \omega_{1} & 1 & \cdots & \cos \left(N_{1}-2\right) \omega_{1} \\
\vdots & \vdots & \vdots & & \vdots \\
\cos N_{1} \omega_{1} & \cos \left(N_{1}-1\right) \omega_{1} & \cos \left(N_{1}-2\right) \omega_{1} & \cdots & 1
\end{array}\right] d \omega_{1}=\left[\Omega_{1 l_{1}}(i, j)\right]
$$

$$
\begin{aligned}
\boldsymbol{c}_{1}^{T} & =\left[\begin{array}{llll}
\cos \left(D_{1}+p_{1}\right) \omega_{1} & \cos \left(D_{1}+p_{1}-1\right) \omega_{1} & \cdots & \cos \left(D_{1}+p_{1}-N_{1}\right) \omega_{1}
\end{array}\right] \\
\boldsymbol{s}_{1}^{T} & =\left[\begin{array}{llll}
\sin \left(D_{1}+p_{1}\right) \omega_{1} & \sin \left(D_{1}+p_{1}-1\right) \omega_{1} & \cdots & \sin \left(D_{1}+p_{1}-N_{1}\right) \omega_{1}
\end{array}\right] \\
\boldsymbol{c}_{2}^{T} & =\left[\begin{array}{llll}
\cos \left(D_{2}+p_{2}\right) \omega_{2} & \cos \left(D_{2}+p_{2}-1\right) \omega_{2} & \cdots & \cos \left(D_{2}+p_{2}-N_{2}\right) \omega_{2}
\end{array}\right] \\
\boldsymbol{s}_{2}^{T} & =\left[\begin{array}{llll}
\sin \left(D_{2}+p_{2}\right) \omega_{2} & \sin \left(D_{2}+p_{2}-1\right) \omega_{2} & \cdots & \sin \left(D_{2}+p_{2}-N_{2}\right) \omega_{2}
\end{array}\right]
\end{aligned}
$$


as the adaptive Simpson's rule or the adaptive Newton-Cotes rule [26]. The matrix $\boldsymbol{U}_{2}$ in (39) can be determined in a similar fashion. Furthermore, matrices $V_{1}$ and $V_{2}$ in (39) are given by

$$
\begin{aligned}
& \boldsymbol{V}_{1}=\int_{-\pi}^{\pi} \int_{0}^{1} W_{1}\left(\omega_{1}\right) W_{3}\left(p_{1}\right) \boldsymbol{p}_{1} \boldsymbol{s}_{1}^{T} d \omega_{1} d p_{1} \\
& \boldsymbol{V}_{2}=\int_{-\pi}^{\pi} \int_{0}^{1} W_{2}\left(\omega_{2}\right) W_{4}\left(p_{2}\right) \boldsymbol{p}_{2} \boldsymbol{s}_{2}^{T} d \omega_{2} d p_{2} .
\end{aligned}
$$

It can be readily shown that

$$
\begin{aligned}
& V_{1}=\mathbf{0} \\
& V_{2}=\mathbf{0} .
\end{aligned}
$$

As a result, we obtain the second term in (28) as

$$
J_{2}\left(\boldsymbol{A}_{1}, \boldsymbol{A}_{2}\right)=\operatorname{tr}\left(\boldsymbol{U}_{1} \boldsymbol{A}_{1}\right) \operatorname{tr}\left(\boldsymbol{U}_{2} \boldsymbol{A}_{2}\right) .
$$

The last term in (28) is a constant

$$
\begin{aligned}
J_{3}= & \int_{\omega_{1}} \int_{\omega_{2}} \int_{p_{1}} \int_{p_{2}} W_{1}\left(\omega_{1}\right) W_{2}\left(\omega_{2}\right) \\
& \cdot W_{3}\left(p_{1}\right) W_{4}\left(p_{2}\right) d \omega_{1} d \omega_{2} d p_{1} d p_{2} \\
= & \text { constant. }
\end{aligned}
$$

Substituting (29), (44), and (45) into (28) leads the final weighted squared-error function to

$$
\begin{aligned}
J\left(\boldsymbol{A}_{1}, \boldsymbol{A}_{2}\right)=\operatorname{tr}\left(\boldsymbol{P}_{1} \boldsymbol{A}_{1}^{T} \Omega_{1} \boldsymbol{A}_{1}\right) \operatorname{tr}\left(\boldsymbol{P}_{2} A_{2}^{T} \boldsymbol{\Omega}_{2} \boldsymbol{A}_{2}\right) \\
+\operatorname{tr}\left(\boldsymbol{U}_{1} \boldsymbol{A}_{1}\right) \operatorname{tr}\left(\boldsymbol{U}_{2} \boldsymbol{A}_{2}\right)+\text { constant. }
\end{aligned}
$$

\section{Closed-Form Solutions for $\boldsymbol{A}_{1}$ AND $\boldsymbol{A}_{2}$}

Our objective here is to find the optimal coefficient matrices $\boldsymbol{A}_{1}$ and $\boldsymbol{A}_{2}$ in (46) such that the error function $J\left(\boldsymbol{A}_{1}, \boldsymbol{A}_{2}\right)$ is minimized. The closed-form optimal solutions can be obtained as follows.

Differentiating the objective function $J\left(\boldsymbol{A}_{1}, \boldsymbol{A}_{2}\right)$ in (46) with respect to the coefficient matrices $A_{1}$ and $\boldsymbol{A}_{2}$, respectively, we obtain

$$
\begin{aligned}
& \frac{\partial J\left(\boldsymbol{A}_{1}, \boldsymbol{A}_{2}\right)}{\partial \boldsymbol{A}_{1}}=2 \boldsymbol{\Omega}_{1} \boldsymbol{A}_{1} \boldsymbol{P}_{1} \operatorname{tr}\left(\boldsymbol{P}_{2} \boldsymbol{A}_{2}^{T} \boldsymbol{\Omega}_{2} \boldsymbol{A}_{2}\right)-2 U_{1}^{T} \operatorname{tr}\left(\boldsymbol{U}_{2} \boldsymbol{A}_{2}\right) \\
& \frac{\partial J\left(\boldsymbol{A}_{1}, A_{2}\right)}{\partial \boldsymbol{A}_{2}}=2 \boldsymbol{\Omega}_{2} \boldsymbol{A}_{2} \boldsymbol{P}_{2} \operatorname{tr}\left(\boldsymbol{P}_{1} \boldsymbol{A}_{1}^{T} \boldsymbol{\Omega}_{1} \boldsymbol{A}_{1}\right)-2 U_{2}^{T} \operatorname{tr}\left(\boldsymbol{U}_{1} \boldsymbol{A}_{1}\right)
\end{aligned}
$$

Setting the above partial derivative matrices to zero results in the matrix equations

$$
\begin{aligned}
& \Omega_{1} \boldsymbol{A}_{1} \boldsymbol{P}_{1} \operatorname{tr}\left(\boldsymbol{P}_{2} A_{2}^{T} \Omega_{2} \boldsymbol{A}_{2}\right)=\boldsymbol{U}_{1}^{T} \operatorname{tr}\left(\boldsymbol{U}_{2} \boldsymbol{A}_{2}\right) \\
& \Omega_{2} \boldsymbol{A}_{2} \boldsymbol{P}_{2} \operatorname{tr}\left(\boldsymbol{P}_{1} \boldsymbol{A}_{1}^{T} \boldsymbol{\Omega}_{1} A_{1}\right)=U_{2}^{T} \operatorname{tr}\left(\boldsymbol{U}_{1} \boldsymbol{A}_{1}\right) .
\end{aligned}
$$

From (48), we obtain

$$
\boldsymbol{A}_{1}=\frac{\left(\boldsymbol{\Omega}_{1}^{-1} \boldsymbol{U}_{1}^{T} \boldsymbol{P}_{1}^{-1}\right) \operatorname{tr}\left(\boldsymbol{U}_{2} \boldsymbol{A}_{2}\right)}{\operatorname{tr}\left(\boldsymbol{P}_{2} A_{2}^{T} \Omega_{2} \boldsymbol{A}_{2}\right)} .
$$

Since $\Omega_{1}$ and $\boldsymbol{P}_{1}$ are symmetric matrices, we have

$$
\boldsymbol{P}_{1} \boldsymbol{A}_{1}^{T} \boldsymbol{\Omega}_{1}=\frac{\boldsymbol{U}_{1} \operatorname{tr}\left(\boldsymbol{U}_{2} A_{2}\right)}{\operatorname{tr}\left(\boldsymbol{P}_{2} \boldsymbol{A}_{2}^{T} \boldsymbol{\Omega}_{2} A_{2}\right)}
$$

Substituting (51) into (49) leads to

$$
\boldsymbol{P}_{2} A_{2}^{T} \Omega_{2} \operatorname{tr}\left(U_{2} A_{2}\right)=U_{2} \operatorname{tr}\left(P_{2} A_{2}^{T} \Omega_{2} A_{2}\right)
$$

which yields

$$
\left(\boldsymbol{P}_{2} \boldsymbol{A}_{2}^{T} \boldsymbol{\Omega}_{2} \boldsymbol{A}_{2}\right) \operatorname{tr}\left(\boldsymbol{U}_{2} \boldsymbol{A}_{2}\right)=\left(\boldsymbol{U}_{2} \boldsymbol{A}_{2}\right) \operatorname{tr}\left(\boldsymbol{P}_{2} \boldsymbol{A}_{2}^{T} \boldsymbol{\Omega}_{2} \boldsymbol{A}_{2}\right)
$$

i.e.,

$$
\boldsymbol{X} \operatorname{tr}(\boldsymbol{Y})=\boldsymbol{Y} \operatorname{tr}(\boldsymbol{X})
$$

where

$$
\begin{aligned}
& X=\boldsymbol{P}_{2} \boldsymbol{A}_{2}^{T} \boldsymbol{\Omega}_{2} \boldsymbol{A}_{2} \\
& \boldsymbol{Y}=\boldsymbol{U}_{2} \boldsymbol{A}_{2} .
\end{aligned}
$$

Hence, if $\operatorname{tr}(\boldsymbol{Y}) \neq 0$, then

$$
\boldsymbol{X}=\alpha \boldsymbol{Y}
$$

where $\alpha$ is a nonzero scalar. Consequently, we have

$$
\begin{aligned}
& A_{1}=\frac{\left(\boldsymbol{\Omega}_{1}^{-1} \boldsymbol{U}_{1}^{T} \boldsymbol{P}_{1}^{-1}\right) \operatorname{tr}\left(\boldsymbol{U}_{2} \boldsymbol{A}_{2}\right)}{\operatorname{tr}\left(\boldsymbol{P}_{2} \boldsymbol{A}_{2}^{T} \boldsymbol{\Omega}_{2} \boldsymbol{A}_{2}\right)}=\frac{\boldsymbol{\Omega}_{1}^{-1} \boldsymbol{U}_{1}^{T} \boldsymbol{P}_{1}^{-1}}{\alpha} \\
& \boldsymbol{A}_{2}=\alpha \boldsymbol{\Omega}_{2}^{-1} \boldsymbol{U}_{2}^{T} \boldsymbol{P}_{2}^{-1} .
\end{aligned}
$$

If $\operatorname{tr}(\boldsymbol{Y})=0$, i.e.,

$$
\operatorname{tr}\left(\boldsymbol{U}_{2} \boldsymbol{A}_{2}\right)=0
$$

we can find an infinite number of $A_{2}$ satisfying (59), but from (50), this leads to

$$
A_{1}=\mathbf{0} \text {. }
$$

Hence, $\operatorname{tr}(\boldsymbol{Y})$ cannot be zero and $A_{1}$ and $\boldsymbol{A}_{2}$ in (57) and (58) are the final optimal coefficient matrices.

Furthermore, although a nonzero scalar $\alpha$ in (57) and (58) can be chosen arbitrarily, in considering the fact that the variable 2-D filter $H\left(z_{1}, z_{2}, p_{1}, p_{2}\right)$ in (2) is the cascade of $H_{1}\left(z_{1}, p_{1}\right)$ and $\mathrm{H}_{2}\left(z_{2}, p_{2}\right)$, it seems reasonable to determine the value of $\alpha$ as follows.

The frequency response of $H_{1}\left(z_{1}, p_{1}\right)$ in $(5)$ at $\left(\omega_{1}, p_{1}\right)=$ $(0,0)$ is

$$
\begin{aligned}
H_{1}(0,0) & =\omega_{10}^{T} \boldsymbol{A}_{1} \boldsymbol{p}_{10} \\
& =\frac{\omega_{10}^{T} \Omega_{1}^{-1} \boldsymbol{U}_{1}^{T} \boldsymbol{P}_{1}^{-1} \boldsymbol{p}_{10}}{\alpha}
\end{aligned}
$$

where

$$
\begin{aligned}
\omega_{10}^{T} & =\left[\begin{array}{llll}
1 & 1 & \cdots & 1
\end{array}\right] \\
\boldsymbol{p}_{10}^{T} & =\left[\begin{array}{llll}
1 & 0 & \cdots & 0
\end{array}\right]
\end{aligned}
$$

and the frequency response of $H_{2}\left(z_{2}, p_{2}\right)$ in (9) at $\left(\omega_{2}, p_{2}\right)=$ $(0,0)$ is

$$
\begin{aligned}
H_{2}(0,0) & =\omega_{20}^{T} \boldsymbol{A}_{2} \boldsymbol{p}_{20} \\
& =\alpha \omega_{20}^{T} \Omega_{2}^{-1} \boldsymbol{U}_{2}^{T} \boldsymbol{P}_{2}^{-1} \boldsymbol{p}_{20}
\end{aligned}
$$

where

$$
\begin{aligned}
& \omega_{20}^{T}=\left[\begin{array}{llll}
1 & 1 & \cdots & 1
\end{array}\right] \\
& \boldsymbol{p}_{20}^{T}=\left[\begin{array}{llll}
1 & 0 & \cdots & 0
\end{array}\right] .
\end{aligned}
$$

It should be noticed that the vectors $\omega_{10}$ and $\omega_{20}, \boldsymbol{p}_{10}$ and $\boldsymbol{p}_{20}$ in (62) and (64) may have different dimensions.

The corresponding 1-D magnitude responses are

$$
\begin{aligned}
& M_{1}(0,0)=\left|H_{1}(0,0)\right| \\
& M_{2}(0,0)=\left|H_{2}(0,0)\right| .
\end{aligned}
$$


If one requires to have $M_{1}(0,0)=M_{2}(0,0)$, then the value of $\alpha$ can be determined using (61) and (63) as

$$
\alpha=\frac{\sqrt{\left|\boldsymbol{\omega}_{10}^{T} \Omega_{1}^{-1} U_{1}^{T} P_{1}^{-1} p_{10}\right|}}{\sqrt{\left|\boldsymbol{\omega}_{20}^{T} \Omega_{2}^{-1} U_{2}^{T} P_{2}^{-1} p_{20}\right|}} .
$$

This $\alpha$ balances the frequency responses of the cascaded 1-D variable transfer functions $H_{1}\left(z_{1}, p_{1}\right)$ and $H_{2}\left(z_{2}, p_{2}\right)$ at $\left(\omega_{1}, \omega_{2}\right)=(0,0)$ and $\left(p_{1}, p_{2}\right)=(0,0)$.

\section{NUMERICAL EXAMPLE}

In this section, we describe a design example to illustrate the proposed method, where the desired variable 2-D FD response (1) is approximated by a 2-D variable FIR filter with the following design parameters:

$$
\begin{aligned}
N_{1} & =N_{2}=35 \\
K_{1} & =K_{2}=5 \\
W_{i}\left(\omega_{i}\right) & = \begin{cases}1 & \omega_{i} \in[0,0.4 \pi), \quad i=1,2 \\
2 & \omega_{i} \in[0.4 \pi, 0.6 \pi) \\
4 & \omega_{i} \in[0.6 \pi, 0.7 \pi) \\
8 & \omega_{i} \in[0.7 \pi, 0.8 \pi) \\
50 & \omega_{i} \in[0.8 \pi, 0.9 \pi] \\
0 & \omega_{i} \in(0.9 \pi, \pi]\end{cases} \\
W_{3}\left(p_{1}\right) & =1, \quad p_{1} \in[0,1] \\
W_{4}\left(p_{2}\right) & =1, \quad p_{2} \in[0,1] .
\end{aligned}
$$

Obviously, the passband of the desired variable 2-D filter is the rectangular range $\left\{\left(\omega_{1}, \omega_{2}\right):\left|\omega_{1}\right| \leq 0.9 \pi,\left|\omega_{2}\right| \leq 0.9 \pi\right\}$ and the remaining region outside the passband is the "don't care" frequency band. Here, the weighting functions $W_{1}\left(\omega_{1}\right), W_{2}\left(\omega_{2}\right)$, $W_{3}\left(p_{1}\right)$, and $W_{4}\left(p_{2}\right)$ are chosen such that the 2-D frequency response of the designed variable filter is almost uniformly flat in the passband. By looking at the values of $W_{1}\left(\omega_{1}\right)$ and $W_{2}\left(\omega_{2}\right)$ we know that larger weighting coefficients are put near the passband edges where the frequency response is difficult to approximate.

From (2) we know that the actual variable 2-D frequency response is given by

$$
\begin{aligned}
H\left(\omega_{1}, \omega_{2},\right. & \left.p_{1}, p_{2}\right) \\
& =H_{1}\left(\omega_{1}, p_{1}\right) H_{2}\left(\omega_{2}, p_{2}\right) \\
& =M_{1}\left(\omega_{1}, p_{1}\right) e^{j \theta_{1}\left(\omega_{1}, p_{1}\right)} M_{2}\left(\omega_{2}, p_{2}\right) e^{j \theta_{2}\left(\omega_{2}, p_{2}\right)} \\
& =M\left(\omega_{1}, \omega_{2}, p_{1}, p_{2}\right) e^{j \theta\left(\omega_{1}, \omega_{2}, p_{1}, p_{2}\right)}
\end{aligned}
$$

where

$$
\begin{aligned}
M\left(\omega_{1}, \omega_{2}, p_{1}, p_{2}\right) & =M_{1}\left(\omega_{1}, p_{1}\right) M_{2}\left(\omega_{2}, p_{2}\right) \\
\theta\left(\omega_{1}, \omega_{2}, p_{1}, p_{2}\right) & =\theta_{1}\left(\omega_{1}, p_{1}\right)+\theta_{2}\left(\omega_{2}, p_{2}\right) .
\end{aligned}
$$

TABLE I

VARIABLE FREQUENCY-RESPONSE ERRORS

\begin{tabular}{c|c|c|c}
\hline$p_{1}$ & $p_{2}$ & $E_{2}\left(p_{1}, p_{2}\right)(\%)$ & $E_{\max }\left(p_{1}, p_{2}\right)$ \\
\hline \hline \multirow{4}{*}{0} & 0 & 0.0280 & 0.0013 \\
\cline { 2 - 4 } & 0.5 & 0.0860 & 0.0016 \\
\cline { 2 - 4 } & 1 & 0.0280 & 0.0013 \\
\hline \multirow{4}{*}{0.5} & 0 & 0.0860 & 0.0016 \\
\cline { 2 - 4 } & 0.5 & 0.1195 & 0.0027 \\
\cline { 2 - 4 } & 1 & 0.0860 & 0.0016 \\
\hline \multirow{4}{*}{1} & 0 & 0.0280 & 0.0013 \\
\cline { 2 - 4 } & 0.5 & 0.0860 & 0.0016 \\
\cline { 2 - 4 } & 1 & 0.0280 & 0.0013 \\
\hline
\end{tabular}

Thus, the actual variable FD responses are given by

$$
\begin{aligned}
\tau_{1}\left(\omega_{1}, \omega_{2}, p_{1}, p_{2}\right) & =-\frac{\partial \theta\left(\omega_{1}, \omega_{2}, p_{1}, p_{2}\right)}{\partial \omega_{1}}-D_{1} \\
& =-\frac{\partial \theta_{1}\left(\omega_{1}, p_{1}\right)}{\partial \omega_{1}}-D_{1} \quad \text { (in } \omega_{1} \text { direction) } \\
\tau_{2}\left(\omega_{1}, \omega_{2}, p_{1}, p_{2}\right) & =-\frac{\partial \theta\left(\omega_{1}, \omega_{2}, p_{1}, p_{2}\right)}{\partial \omega_{2}}-D_{2} \\
& =-\frac{\partial \theta_{2}\left(\omega_{2}, p_{2}\right)}{\partial \omega_{2}}-D_{2} \quad \text { (in } \omega_{2} \text { direction). }
\end{aligned}
$$

Therefore, the variable fractional delays in $\omega_{1}$ and $\omega_{2}$ directions are completely determined by the phase responses of $H_{1}\left(z_{1}, p_{1}\right)$ and $H_{2}\left(z_{2}, p_{2}\right)$, respectively. That is, the function $\tau_{1}\left(\omega_{1}, \omega_{2}, p_{1}, p_{2}\right)$ only depends on the variables $\omega_{1}$ and $p_{1}$, and the function $\tau_{2}\left(\omega_{1}, \omega_{2}, p_{1}, p_{2}\right)$ only depends on the variables $\omega_{2}$ and $p_{2}$. To evaluate the design performance, the normalized root-mean-squared (RMS) error of the variable 2-D frequency response in (71), found at the bottom of the page, and the maximum absolute frequency-response error

$$
\begin{array}{r}
E_{\max }\left(p_{1}, p_{2}\right)=\max \left\{\left|H\left(\omega_{1}, \omega_{2}, p_{1}, p_{2}\right)-H_{d}\left(\omega_{1}, \omega_{2}, p_{1}, p_{2}\right)\right|,\right. \\
\left.\left|\omega_{1}\right| \leq 0.9 \pi,\left|\omega_{2}\right| \leq 0.9 \pi\right\} \quad \text { (72) }
\end{array}
$$

are used, where $E_{2}\left(p_{1}, p_{2}\right)$ and $E_{\max }\left(p_{1}, p_{2}\right)$ are the functions of $p_{1}$ and $p_{2}$. Table I lists the normalized RMS errors $E_{2}\left(p_{1}, p_{2}\right)$ and the maximum absolute errors $E_{\max }\left(p_{1}, p_{2}\right)$ for some fixed $\left(p_{1}, p_{2}\right)$ values. From Table I, we can find that the normalized RMS errors are very small, and the maximum absolute errors are about 0.002 . In addition, we can also find that both the RMS error and the maximum absolute error for $\left(p_{1}, p_{2}\right)=(0.5,0.5)$ are the largest among all the combinations of $p_{1}$ and $p_{2}$. Fig. 1 shows the 2-D magnitude response for $\left(p_{1}, p_{2}\right)=(0.5,0.5)$. We can observe that the magnitude response in the passband is extremely flat $(\approx 1)$. Fig. 2 shows the absolute errors of the

$$
E_{2}\left(p_{1}, p_{2}\right)=\frac{\left[\int_{-0.9 \pi}^{0.9 \pi} \int_{-0.9 \pi}^{0.9 \pi}\left|H\left(\omega_{1}, \omega_{2}, p_{1}, p_{2}\right)-H_{d}\left(\omega_{1}, \omega_{2}, p_{1}, p_{2}\right)\right|^{2} d \omega_{1} d \omega_{2}\right]^{1 / 2}}{\left[\int_{-0.9 \pi}^{0.9 \pi} \int_{-0.9 \pi}^{0.9 \pi}\left|H_{d}\left(\omega_{1}, \omega_{2}, p_{1}, p_{2}\right)\right|^{2} d \omega_{1} d \omega_{2}\right]^{1 / 2}} \times 100 \%
$$




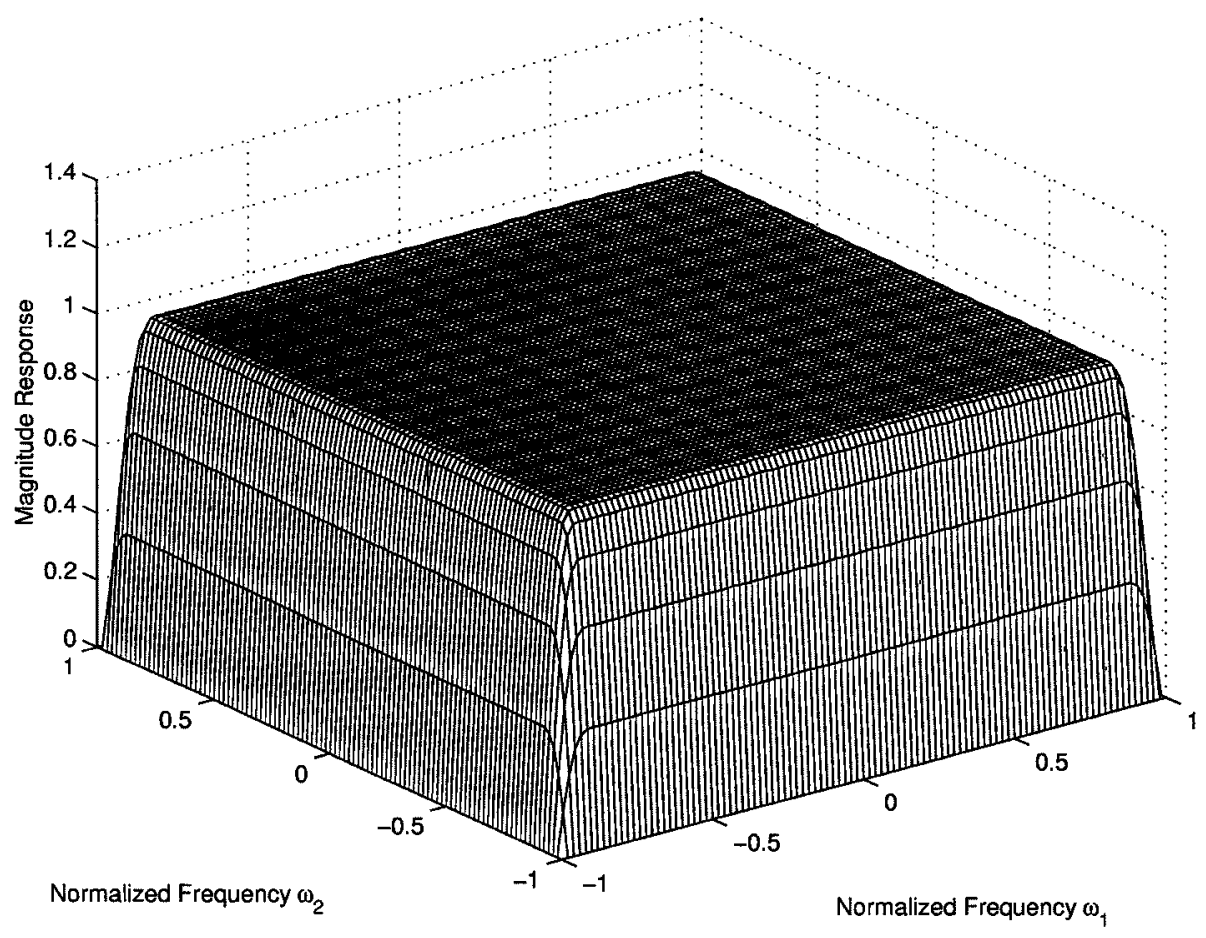

Fig. 1. 2-D magnitude response for $\left(p_{1}, p_{2}\right)=(0.5,0.5)$.

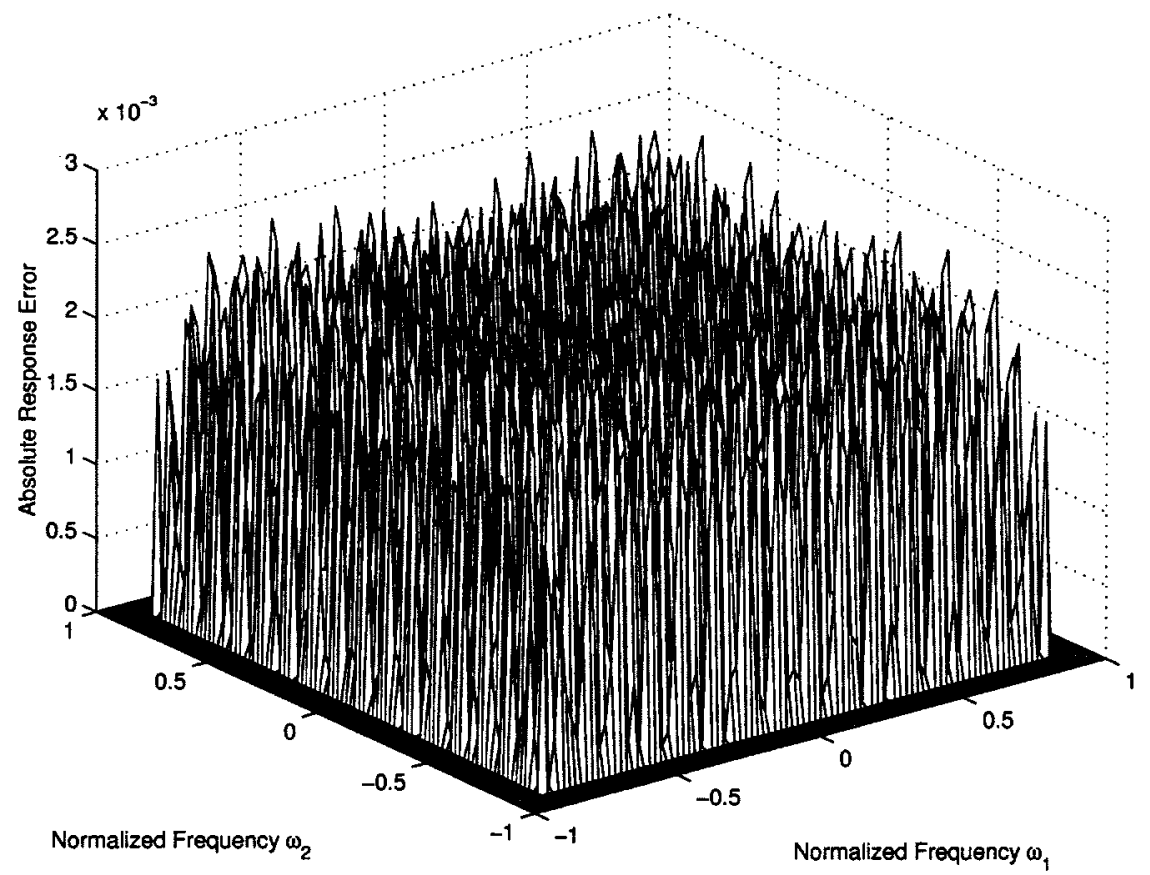

Fig. 2. Absolute frequency response error in the passband for $\left(p_{1}, p_{2}\right)=(0.5,0.5)$.

2-D frequency response in the passband, which are fairly small. In addition, since we put the weighting functions $W_{1}\left(\omega_{1}\right)=$ $W_{2}\left(\omega_{2}\right)=0$ in the "don't care" band where $\omega_{1}, \omega_{2} \in(0.9 \pi, \pi]$, the magnitude response in the "don't care" band does not have to be flat, which can be observed from Fig. 1. This is based on the assumption that the 2-D signal to be processed has frequency components only within the passband. However, if the 2-D signal includes extra high-frequency components such as noise, the FD filter designed here has to work in cascade with other standard filters such as a low-pass filter to perform frequency selecting operations as mentioned in [27].

Fig. 3 illustrates the FD response $\tau_{1}\left(\omega_{1}, \omega_{2}, p_{1}, p_{2}\right)$ in the passband, which is the function of only $\omega_{1}$ and $p_{1}$, and Fig. 4 shows the corresponding absolute deviation of $\tau_{1}\left(\omega_{1}, \omega_{2}, p_{1}, p_{2}\right)$ from the desired FD response. The normalized RMS error of $\tau_{1}\left(\omega_{1}, \omega_{2}, p_{1}, p_{2}\right)$ is $0.5063 \%$, and the maximum absolute error is 0.0135 . Moreover, Figs. 5 and 6 illustrate the passband FD responses $\tau_{2}\left(\omega_{1}, \omega_{2}, p_{1}, p_{2}\right)$ and 


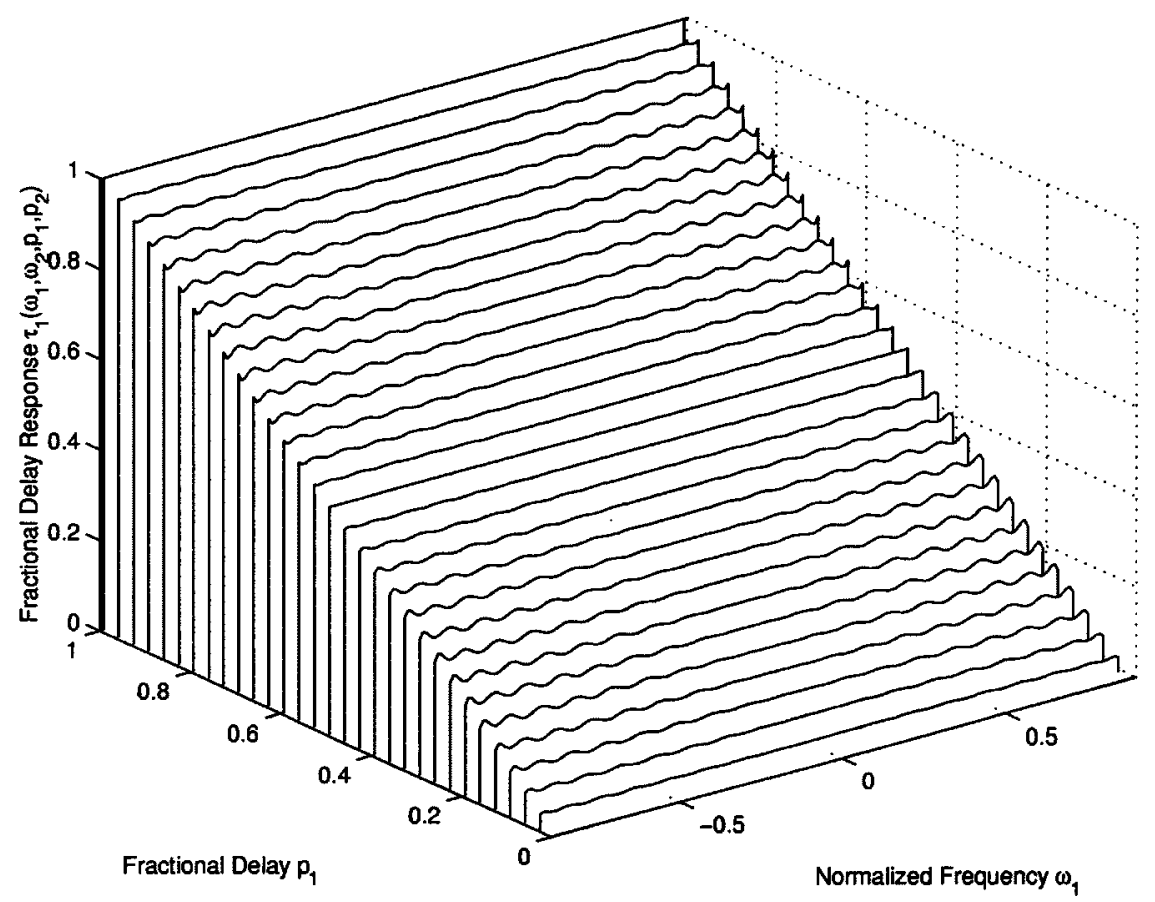

Fig. 3. Variable fractional delay response $\tau_{1}\left(\omega_{1}, \omega_{2}, p_{1}, p_{2}\right)$.

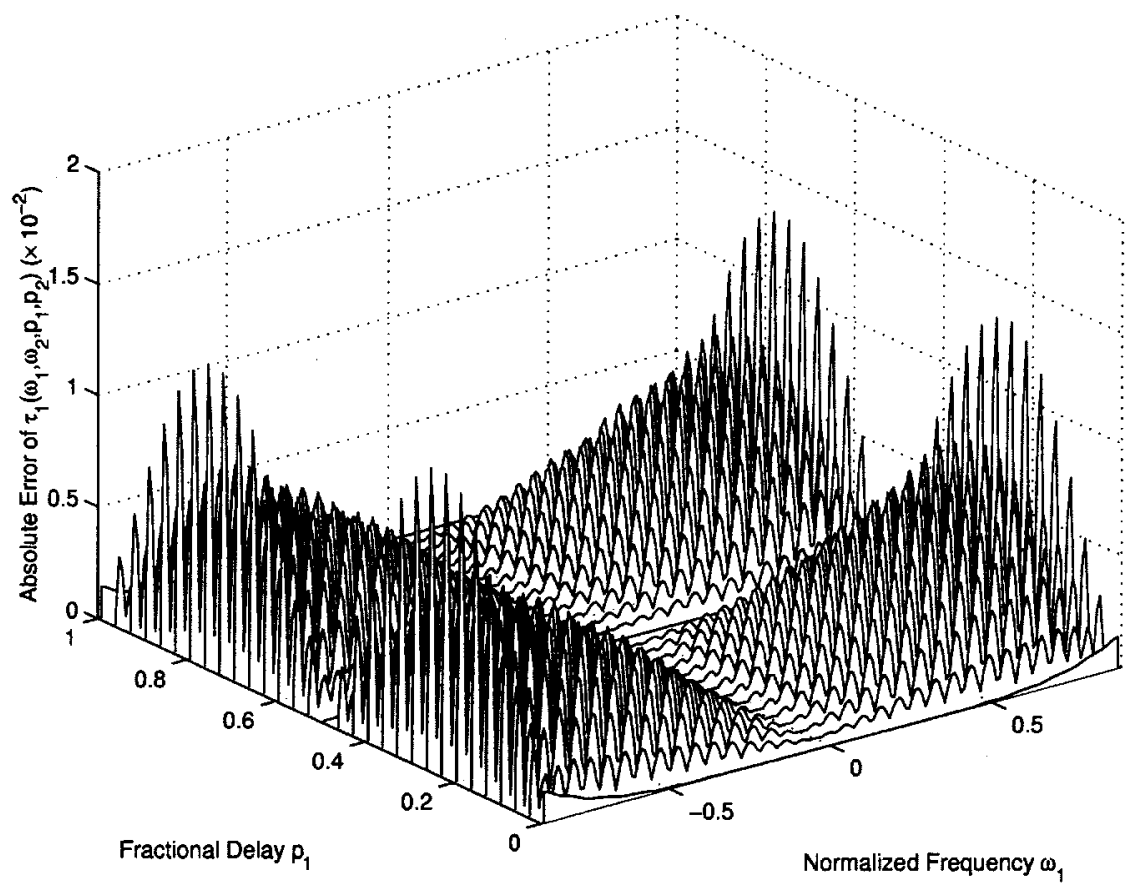

Fig. 4. Absolute error of variable fractional delay $\tau_{1}\left(\omega_{1}, \omega_{2}, p_{1}, p_{2}\right)$.

the corresponding absolute errors. The normalized RMS error and the maximum absolute deviation are the same as those of $\tau_{1}\left(\omega_{1}, \omega_{2}, p_{1}, p_{2}\right)$. From the above results, we observe that the variable FD responses in the passband are quite flat.

MATLAB version 5.2 was used in the computer simulations. The integral (42) is evaluated by using MATLAB command $\{\backslash \mathrm{tt}\{$ quad8\}/tt $\}$ which employs adaptive Newton-Cotes panel rule. In order to avoid numerical ill-conditioning problems which arise in computing the inverses of the matrices $\Omega_{1}, \boldsymbol{P}_{1}$, $\Omega_{2}$, and $P_{2}$ in (57) and (58), they are first decomposed by using Cholesky decomposition as

$$
\begin{array}{ll}
\boldsymbol{\Omega}_{1}=\boldsymbol{R}_{1}^{T} \boldsymbol{R}_{1}, & \boldsymbol{P}_{1}=\boldsymbol{S}_{1}^{T} \boldsymbol{S}_{1} \\
\boldsymbol{\Omega}_{2}=\boldsymbol{R}_{2}^{T} \boldsymbol{R}_{2}, & \boldsymbol{P}_{2}=S_{2}^{T} S_{2}
\end{array}
$$




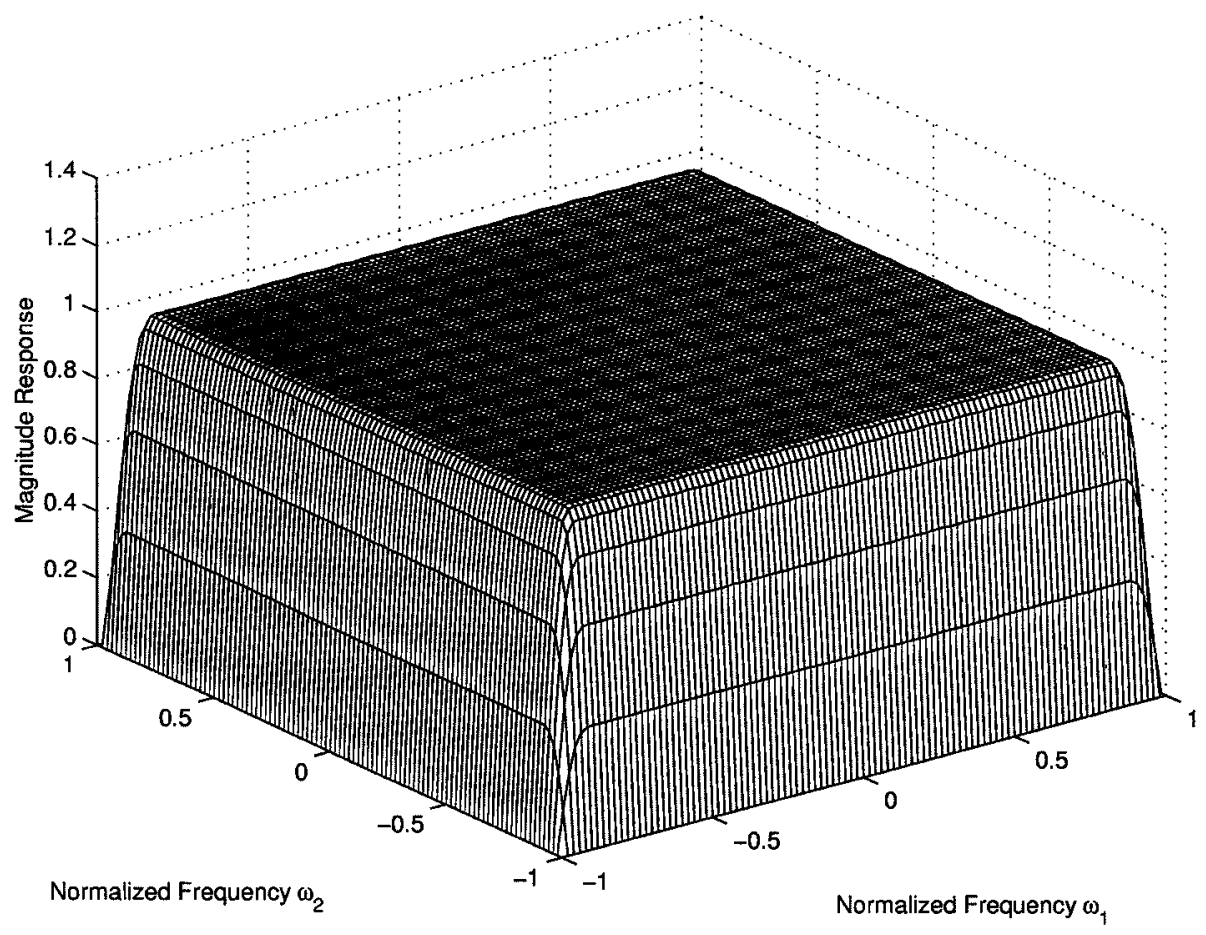

Fig. 5. Variable fractional delay response $\tau_{2}\left(\omega_{1}, \omega_{2}, p_{1}, p_{2}\right)$.

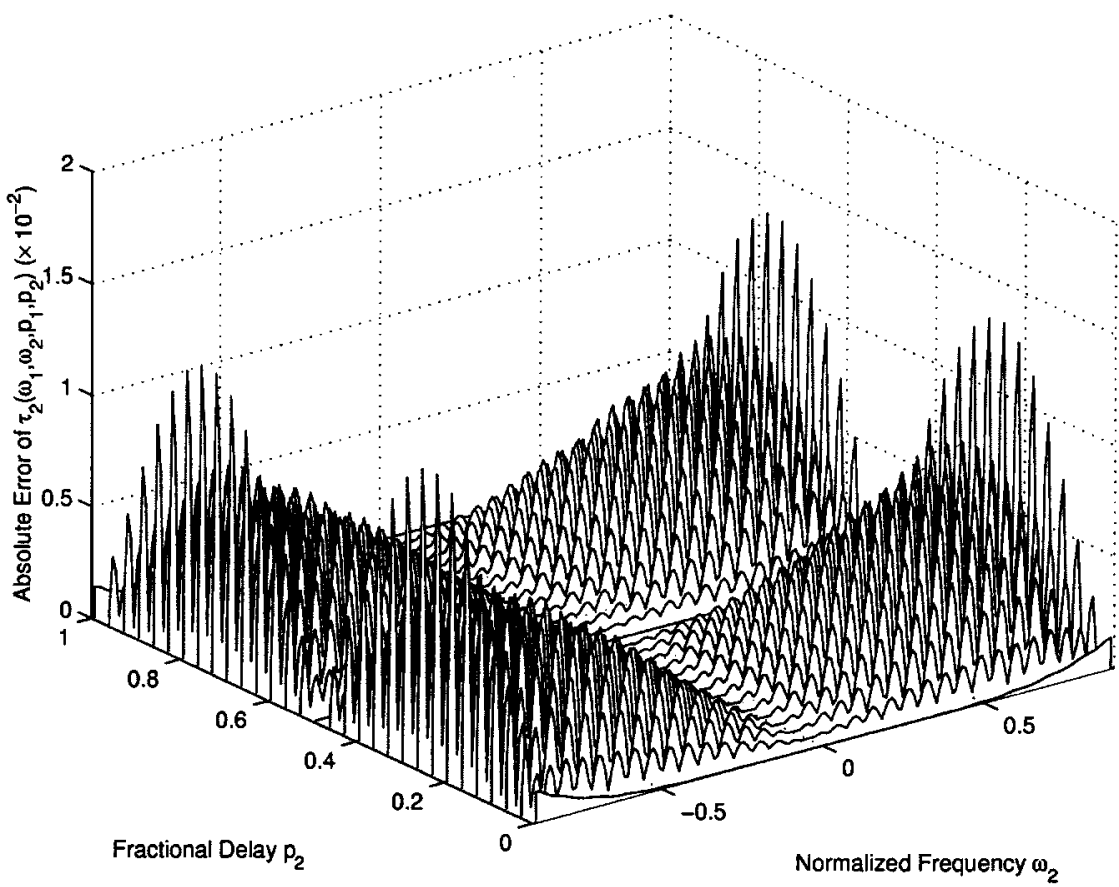

Fig. 6. Absolute error of variable fractional delay $\tau_{2}\left(\omega_{1}, \omega_{2}, p_{1}, p_{2}\right)$.

where $\boldsymbol{R}_{1}, \boldsymbol{S}_{1}, \boldsymbol{R}_{2}$, and $\boldsymbol{S}_{2}$ are upper triangular matrices. Then equations (57) and (58) are rearranged as

$$
\begin{gathered}
\boldsymbol{A}_{1}=\frac{\boldsymbol{R}_{1}^{-1}\left[\boldsymbol{R}_{1}^{-T}\left(\boldsymbol{U}_{1}^{T} \boldsymbol{S}_{1}^{-1}\right) \boldsymbol{S}_{1}^{-T}\right]}{\alpha} \\
A_{2}=\alpha \boldsymbol{R}_{2}^{-1}\left[\boldsymbol{R}_{2}^{-T}\left(\boldsymbol{U}_{2}^{T} \boldsymbol{S}_{2}^{-1}\right) \boldsymbol{S}_{2}^{-T}\right] .
\end{gathered}
$$

The groupings in (74) and (75) assure the numerically stable solutions because of the following reasons.
1) The decompositions (73) imply that the inverses of the matrices $\Omega_{1}, \boldsymbol{P}_{1}, \Omega_{2}$, and $\boldsymbol{P}_{2}$ can be indirectly calculated as

$$
\begin{array}{ll}
\Omega_{1}^{-1}=R_{1}^{-1} R_{1}^{-T}, & P_{1}^{-1}=S_{1}^{-1} S_{1}^{-T} \\
\Omega_{2}^{-1}=R_{2}^{-1} R_{2}^{-T}, & P_{2}^{-1}=S_{2}^{-1} S_{2}^{-T}
\end{array}
$$

where the condition numbers of $\boldsymbol{R}_{1}, \boldsymbol{S}_{1}, \boldsymbol{R}_{2}$, and $\boldsymbol{S}_{2}$ are much smaller than those of $\boldsymbol{\Omega}_{1}, \boldsymbol{P}_{1}, \boldsymbol{\Omega}_{2}$, and $\boldsymbol{P}_{2}$, thus more accurate inversions can be obtained. 
2) The entries of $\boldsymbol{U}_{1}$ and $\boldsymbol{U}_{2}$ are very small in magnitude, thus the groupings $\boldsymbol{U}_{1}^{T}$ with $\boldsymbol{S}_{1}^{-1}$ and $\boldsymbol{U}_{2}^{T}$ with $\boldsymbol{S}_{2}^{-1}$ make it possible for the small entries in $\boldsymbol{U}_{1}^{T}$ and $\boldsymbol{U}_{2}^{T}$ to cancel out the large entries in $\boldsymbol{S}_{1}^{-1}$ and $\boldsymbol{S}_{2}^{-1}$, respectively. This in turn results in the desired numerical stability. It was found that other matrix decomposition methods such as LU, SVD, and QR methods can also be utilized to decompose $\boldsymbol{\Omega}_{1}, \boldsymbol{P}_{1}, \Omega_{2}$, and $\boldsymbol{P}_{2}$ to assure the similar numerical stability, but Cholesky decomposition requires slightly reduced computational complexity compared to the other methods.

Finally, we would like to summarize the whole design process stated in the preceding sections and comment on the implementation of the resulting 2-D variable filter as follows. First, the design can be performed by the following.

Design-Step-1: Compute matrices $\boldsymbol{P}_{1}, \boldsymbol{\Omega}_{1}$, and $\boldsymbol{U}_{1}$ by using formulae (30), (33), and (40). The matrices $\boldsymbol{P}_{2}, \Omega_{2}$, and $U_{2}$ can be computed in the same way.

Design-Step-2: Determine the scaling factor $\alpha$ in (65).

Design-Step-3: Perform Cholesky decompositions on matrices $\boldsymbol{\Omega}_{1}, \boldsymbol{P}_{1}$, and $\boldsymbol{\Omega}_{2}, \boldsymbol{P}_{2}$ as (73).

Design-Step-4: Compute the optimal coefficient matrices $A_{1}$ and $A_{2}$ as (74) and (75).

The above four steps complete the off-line design of the variable 2-D FIR filter.

Second, once the optimal coefficient matrices $A_{1}$ and $A_{2}$ are found, the resulting variable filter can be applied to real-time tuning for any given values of the fractional delays $p_{1}$ and $p_{2}$. This can be seen from (5) and (9), which only need matrix multiplications $A_{1} \boldsymbol{p}_{1}$ and $A_{2} p_{2}$ to obtain the new 2-D filter coefficients for some given values of $p_{1}$ and $p_{2}$. Another efficient method for the real-time implementation of the resulting 2-D variable FIR filter (2) is to implement $H_{1}\left(z_{1}, p_{1}\right)$ and $H_{2}\left(z_{2}, p_{2}\right)$, respectively, by using the Farrow structure [16], then $H_{1}\left(z_{1}, p_{1}\right)$ and $H_{2}\left(z_{2}, p_{2}\right)$ are connected in cascade to obtain $H\left(z_{1}, z_{2}, p_{1}, p_{2}\right)$.

\section{CONCLUSION}

We have proposed a weighted least-squares method for designing variable 2-D FIR digital filters with continuously variable FD responses. The design is formulated as a weighted leastsquares minimization problem without sampling the 2-D frequencies $\omega_{1}, \omega_{2}$, and variable 2-D fractional delays $p_{1}, p_{2}$, thus the final design accuracy is not affected by the sampling grid densities. Furthermore, since no discretizations of the parameters are required in computing the closed-form optimal coefficient matrices, the computational complexity is significantly reduced.

A design example has been given to illustrate the proposed method. This method may also be generalized to deal with higher dimensional $(\geq 3)$ cases, however the derivations may become far more involved, and further studies need to be carried out for developing tractable design algorithms.

\section{ACKNOWLEDGMENT}

The authors thank the anonymous reviewers for their constructive comments and valuable suggestions.

\section{REFERENCES}

[1] P. Jarske, Y. Neuvo, and S. K. Mitra, "A simple approach to the design of linear phase FIR digital filters with variable characteristics," Signal Processing, vol. 14, no. 4, pp. 313-326, June 1988.

[2] L. J. Karam and J. H. McClellan, "Efficient design of families of FIR filters by transformations," in Proc. IEEE Int. Conf. Acoustics, Speech and Signal Processing (ICASSP'96), vol. 3, May 1996, pp. 1359-1362.

[3] — , "Efficient design of digital filters for 2-D and 3-D depth migration," IEEE Trans. Signal Processing, vol. 45, pp. 1036-1044, Apr 1997.

[4] T.-B. Deng, "Variable 2-D FIR digital filter design and parallel implementation," IEEE Trans. Circuits Syst. II, vol. 46, pp. 631-635, May 1999.

[5] R. Zarour and M. M. Fahmy, "A design technique for variable digital filters," IEEE Trans. Circuits Syst., vol. 36, pp. 1473-1478, Nov. 1989.

[6] T.-B. Deng, "Design of recursive 1-D variable filters with guaranteed stability," IEEE Trans. Circuits Syst. II, vol. 44, pp. 689-695, Sept. 1997.

[7] R. Zarour and M. M. Fahmy, "A design technique for variable two-dimensional recursive digital filters," Signal Processing, vol. 17, no. 2, pp. 175-182, June 1989.

[8] T.-B. Deng, "Design of variable 2-D linear phase recursive digital filters with guaranteed stability," IEEE Trans. Circuits Syst. I, vol. 45, pp. 859-863, Aug. 1998.

[9] _ - "Design of linear phase variable 2-D digital filters using real-complex decomposition," IEEE Trans. Circuits Syst. II, vol. 45, pp. 330-339, Mar. 1998.

[10] G. Stoyanov and M. Kawamata, "Variable digital filters," J. Signal Processing, vol. 1, no. 4, pp. 275-289, July 1997.

[11] F. M. Gardner, "Interpolation in digital modems-Part I: Fundamentals," IEEE Trans. Commun., vol. 41, pp. 502-508, Mar. 1993.

[12] L. Erup, F. M. Gardner, and F. A. Harris, "Interpolation in digital modems-Part II: Implementation and performance," IEEE Trans. Commun., vol. 41, pp. 998-1008, June 1993.

[13] V. Valimaki, T. I. Laakso, and J. Mackenzie, "Elimination of transient in time-varying allpass fractional delay filters with application to digital waveguide modeling," in Proc. Int. Computer Music Conf. (ICMC-95), Banff, Canada, Sept. 1995, pp. 327-334

[14] V. Valimaki, "Discrete-Time Modeling of Acoustic Tubes Using Fractional Delay Filters," Doctoral, Helsinki Univ. Technol., Faculty of Electrical Eng., Laboratory of Acoustics and Audio Signal Processing, Espoo, Finland, Dec. 1995.

[15] T. I. Laakso, V. Valimaki, M. Karjalainen, and U. K. Laine, "Splitting the unit delay: Tools for fractional delay filter design," IEEE Signal Processing Mag., vol. 13, pp. 30-60, Jan. 1996.

[16] C. W. Farrow, "A continuously variable digital delay elements," in Proc. 1988 IEEE Int. Symp. Circuits and Systems (ISCAS'88), vol. 3, Espoo, Finland, June 1988, pp. 2641-2645.

[17] P. J. Kootsookos and R. C. Williamson, "FIR approximation of fractional sample delay systems," IEEE Trans. Circuits Syst. II, vol. 43, pp. 269-271, Mar. 1996.

[18] A. Tarczynski, G. D. Cain, E. Hermanowicz, and M. Rojewski, "WLS design of variable frequency response FIR filters," in Proc. 1997 IEEE Int. Symp. Circuits and Systems (ISCAS'97), Hong Kong, June 1997, pp. 2244-2247.

[19] W.-S. Lu and T.-B. Deng, "An improved weighted least-squares design of FIR digital filters with variable fractional delay," in Proc. 1999 IEEE Int. Symp. Circuits and Systems (ISCAS'99), vol. III, FL, May -June 1999, pp. 383-386.

[20] H. S. Hou and H. C. Andrews, "Cubic splines for image interpolation and digital filtering," IEEE Trans. Acoust., Speech, Signal Processing, vol. ASSP-26, pp. 508-517, Dec. 1978

[21] R. G. Keys, "Cubic convolution intgerpolation for digital image processing," IEEE Trans. Acoust., Speech, Signal Processing, vol. ASSP-29, pp. 1153-1160, Dec. 1981

[22] J. A. Park, R. V. Kenyon, and D. E. Troxel, "Comparison of interpolation methods for image resampling," IEEE Trans. Med. Imag., vol. 2, pp. 31-39, Mar. 1983.

[23] E. Maeland, "On the comparison of interpolation methods," IEEE Trans. Med. Imag., vol. MI-7, pp. 213-217, Sept. 1988.

[24] N. A. Dodgson, "Quadratic interpolation for image resampling," IEEE Trans. Image Processing, vol. 6, pp. 1322-1326, Sept. 1997.

[25] R. A. Horn and C. R. Johnson, Matrix Analysis. Cambridge, U.K.: Cambridge Univ. Press, 1985

[26] W. H. Press, S. A. Teukolsky, W. T. Vetterling, and B. P. Flannery, Numerical Recipes in C, 2nd ed. Cambridge, U.K.: Cambridge Univ. Press, 1992. 
[27] E. Hermanowicz, M. Rojewski, G. D. Cain, and A. Tarczynski, "Special discrete-time filters having fractional delay," Signal Processing, vol. 67, no. 3, pp. 279-289, June 1998 .

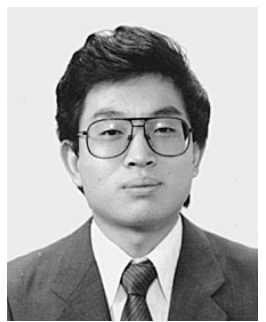

Tian-Bo Deng (M'92-SM'99) received the Ph.D. degree in electronic engineering from Tohoku University, Japan, in 1991.

He was with the Department of Information and Computer Sciences, Toyohashi University of Technology, Japan, as a Research Associate from 1991 to 1992. In 1992, he was selected by the Japanese Government as a Special Researcher for carrying out the Basic Science Program in the Insitute of Physical and Chemical Research. He joined the Department of Information Science, Faculty of Science, Toho University, as an Assistant Professor from 1994 to 1998, and is presently an Associate Professor. From 1998 to 1999, he was also a Visiting Professor in the Department of Electrical and Computer Engineering, University of Victoria, Canada. His research interests include control theory, speech processing, design theory of constant multidimensional digital filters, and design theory of variable one-dimensional and multidimensional digital filters.

Dr. Deng is a Member of the Institute of Electronics, Information and Communication Engineers (IEICE), Japan, and the European Association for Speech, Signal and Image Processing (EURASIP).

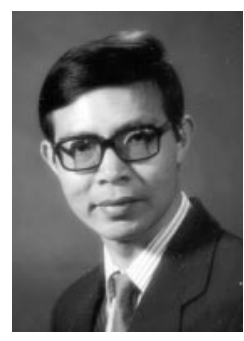

Wu-Sheng Lu (S'81-M'85-SM'90-F'99) received the B.S. degree in mathematics from Fudan University, China, in 1964, the M.S. degree in electrical engineering, and the Ph.D. degree in control science from the University of Minnesota, Minneapolis, in 1983, and 1984, respectively.

He was a post-doctoral Fellow at the University of Victoria, Victoria, BC, Canada, in 1985 and a Visiting Assistant Professor at the University of Minnesota in 1986. Since 1987, he has been with the University of Victoria, where he is currently a Professor. His teaching and research interests are in the areas of digital signal processing and numerical optimization. He is the co-author, with A. Antoniou, of Two-Dimensional Digital Filters (Marcel Dekker: 1992).

Dr. Lu served as an Associate Editor in 1989 and then Editor from 1990 to 1992 of the Canadian Journal of Electrical and Computer Engineering. He was an Associate Editor of IEEE TRANSACTIONS ON CIRCUITS AND SYSTEMS II from 1993 to 1995. Presently, he serves as an Associate Editor for the IEEE TRANSACTIONS ON CIRCUITS AND SYSTEMS and the International Journal of Multidimensional Systems and Signal Processing. 